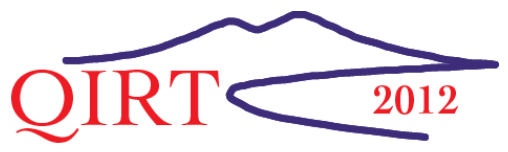

\title{
Development of an infrared system coupled with a weather station for real time atmospheric corrections using GPU computing: Application to bridge monitoring
}

by J. Dumoulin* and R. Averty*

*LUNAM Université, IFSTTAR, MACS, F-44340, Bouguenais, France, jean.dumoulin@ifsttar.fr

\section{Abstract}

A system (software and hardware) has been studied and developed to allow long term monitoring of transport infrastructures in a stand alone configuration. It is based on the implementation of low cost infrared cameras available on the market coupled with other measurement systems to feed simplified radiative models running on GPU available on small PC. Results obtained after implementation on a bridge open to traffic are presented and analysed.

\section{Introduction}

One of the objectives of ISTIMES project is to evaluate the potentialities offered by the integration of different electromagnetic techniques able to perform non-invasive diagnostics for surveillance and monitoring of transport infrastructures. Among the EM (Electromagnetic) methods investigated, uncooled infrared camera is a promising technique due to its dissemination potential according to its low cost on the market.

Infrared thermography, when it is used in quantitative mode (not in laboratory conditions) and not in qualitative mode (vision applied to survey), requires to process in real time thermal radiative corrections on raw data acquired to take into account influences of natural environment evolution with time. But, camera sensor has to be enough smart to apply in real time calibration law and radiometric corrections in a varying atmosphere. So the work presented hereafter deals with a measurement system studied and developed with low cost infrared cameras available on the market coupled with other sensors to feed simplified radiative models running, in real time, on GPU available on small PC.

\section{Measurement system developed}

The system studied and developed uses a fast Ethernet camera FLIR A320 [1] coupled with a VAISALA WXT520 [2] weather station and a light GPS unit [3] for positioning and timing. Figure 1 (left) shows a schematic representation of the interconnection between all measurement systems.
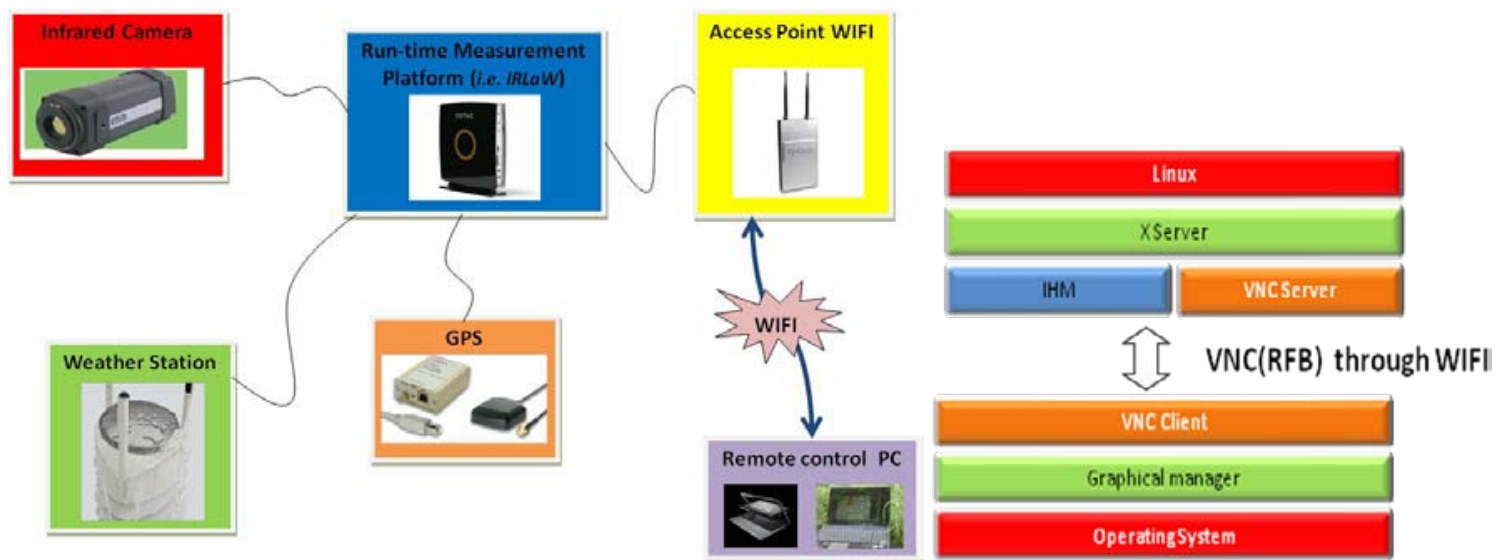

Fig. 1. Schematic representation of: the measurement system developed (left) and the principle of interoperability (right)

The measurement system can be remotely control via WiFi connection. For field application an outdoor WiFi access Point is used to improve performances. Though the run time measurement platform is running under Linux the remote control PC that can be used can be operated on different operating system (from PC to Mac up to android smart phone). In the present study a VNC (Virtual Network Computing) Server was mounted on the measurement platform and a VNC Client was used under a remote PC running a MSWindows operating system. To facilitate interoperability at ground level on field a tactile tablet PC was used for control operations and setting the system parameters at the beginning of the experiments. Figure 1 right shows a scheme of the wireless remote connection principle with VNC that uses RFB (Remote Frame Buffer) protocol. 
The HMI (Human Machine Interface) was developed under Linux using OpenSource and complementary elements of software developed at IFSTTAR. This new HMI called "IrLaW" (Infrared thermography through Lan and WiFi) has various functionalities that let it compliant to be use both for NDT experiments in laboratory and also in real site for long term monitoring. It can be used with other Ethernet infrared cameras (i.e. visible ones). To introduce new camera, access to measured data at raw level and camera parameter elementary controlled commands are required to maintain "pseudo" real time capabilities (no real time scheduler). For the infrared camera used in the present study, it has been made possible thanks to a specific agreement signed with FLIR Company.

Figure 2 shows a schematic representation of the developed application and its interconnection with the infrared camera, weather station and GPS unit for long term monitoring application on real field.

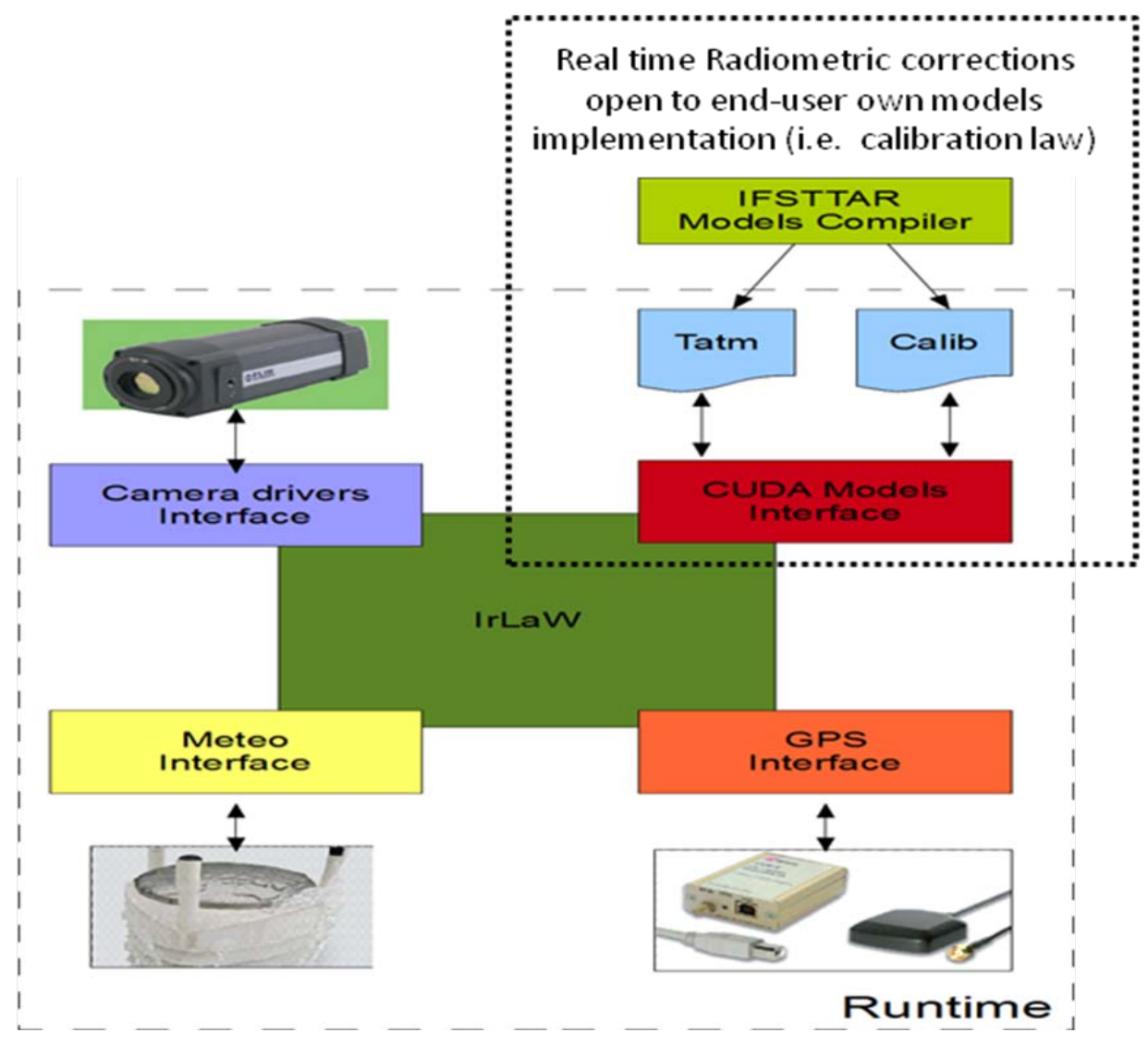

Fig. 2. Schematic representation of IrLaW measurement main functionalities

The prototype system studied and developed is implemented on low cost small computer that integrates a GPU card to allow real time parallel computing [4]. Measurement corrections are carried out inline using weather station measurements. The IFSTTAR models compiler allows one to use its own calibration and measurement correction laws or the ones proposed by infrared camera provider.

To tackle the objective of in line real time temperature conversion from radiative heat flux measured, a set of mathematical function that can be used to build radiative corrections and calibration law models have been developed. One can generate its own model using standard mathematical functions.

Main elements of the model compilator language are reported hereafter:

- $\quad$ Key words (i.e. math function): declare, set, sqrt, exp, log, pow, add, sub, div, mul, neg, print, return

- $\quad$ Operators: $+,-, /{ }^{*},{ }^{\prime},{ }^{\wedge}$

- Temporal marks:

- Brackets are used and mandatory to fix calculus priorities,

- Comments are notified at the beginning of a line by using the // symbol,

- Each instruction ends by semi colon.

- $\quad$ Numerical data only in real format.

- Variables: 32 characters

Then models are compiled to produce libraries. These libraries are used with a high level API hiding internals. IrLaW takes benefit of these libraries for making apparent temperature data conversion from raw data and offer the 
possibility to use new models without any modification of the original software. Compiled models, thanks to our developed compilation tool, are directly tuned (two librairies are generated) to run on CPU or on GPU (CUDA compliant), depending on PC used.

Finally, thanks to GPU parallel computing all models are operated at pixel level, that allows calculation for instance with emissivity matrix and distance matrix (between sensor and 3D survey scene). In practise, models are written in a text file, compiled and after copying it into a dedicate directory, they are then ready to use by calling it through the associated combo box of the HMI.

Figure 3 shows a schematic representation of infrared raw data matrix conversion into infrared image of apparent surface temperature using models.

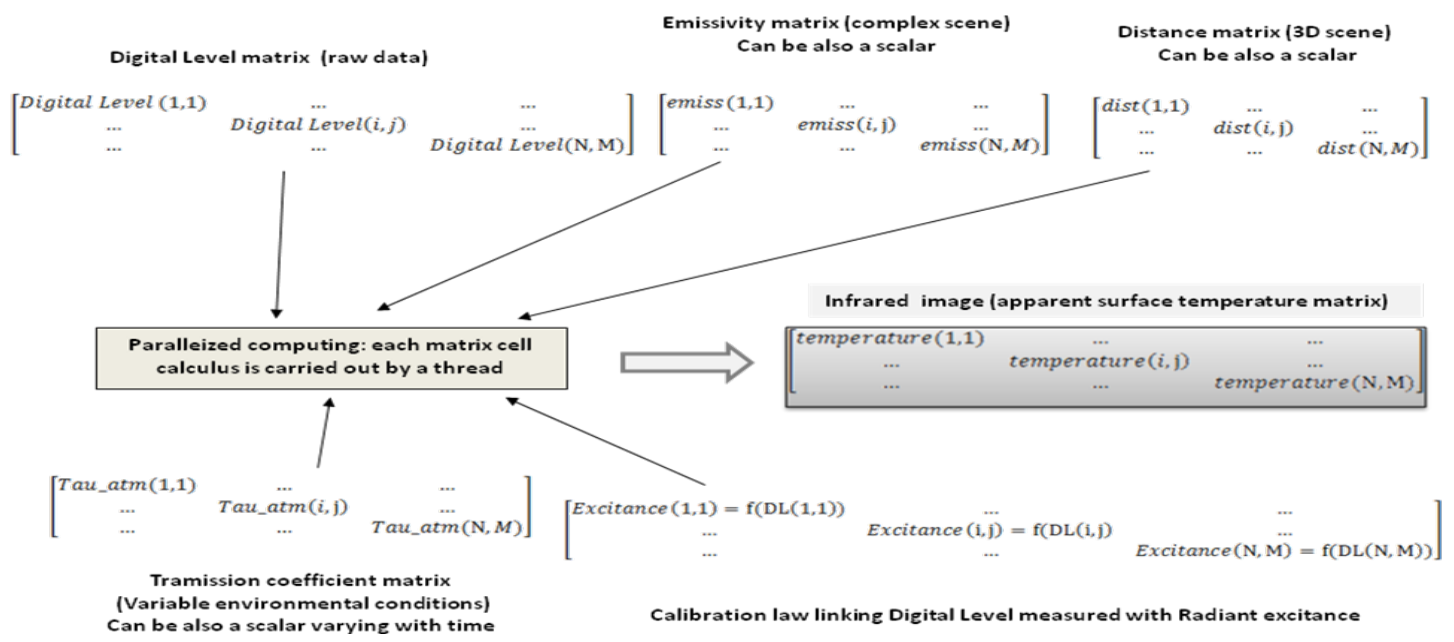

Fig. 3. Schematic of representation of the hardware

Tests made with a mini-PC using CUDA GPU show that we can gain 40 times and more performance ratio versus standard CPU (computing and displaying thermal images in the same time).

In the present study, classical radiative heat balance [5] as shown in Figure 4 was used.

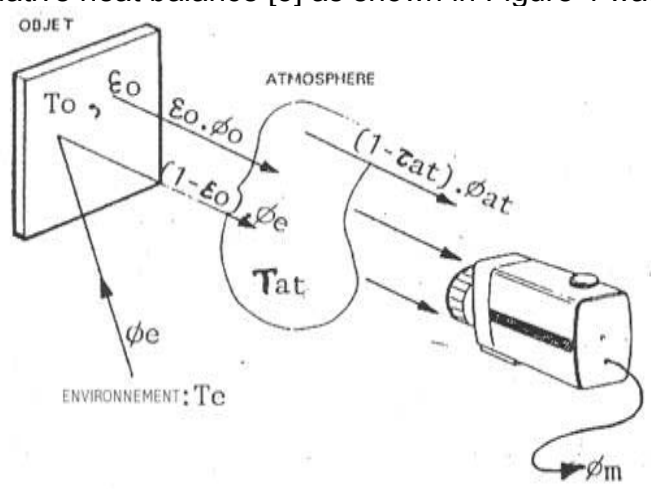

Fig. 4. Schematic representation of the hardware

The simplified radiometric [5] heat balance Eq. (1) is calculated at each time step of measurement using information measured by the weather station coupled with a atmospheric transmission model.

$$
\mathrm{DL}_{0}^{\prime}=\tau_{\mathrm{atm}} \varepsilon_{0} \mathrm{DL}_{0}+\tau_{\mathrm{atm}}\left(1-\varepsilon_{0}\right) \mathrm{DL}_{\mathrm{e}}+\left(1-\tau_{\mathrm{atm}}\right) \mathrm{DL}_{\mathrm{atm}}
$$

With: $\tau_{\mathrm{atm}}$ the atmospheric transmission computed, $\varepsilon_{0}$ the measured object emissivity and DL the digital level at detector outlet level.

IrLaw interface software interface is presented in Figure 5. It has standard functionalities that allow image averaging, fixing the number of frame by thermal image sequence saved, changing the sampling frequency during an acquisition with no interruption. Scenarios are written using $\mathrm{xml}$ formalism and several predefined functions are 
accessible. For field application, scenarios were implemented to generate automatically average image at a fixed frequency (while camera was running at higher frequency) and thermal image sequence were saved at fixed time period to avoid loss of data in case of malfunction.
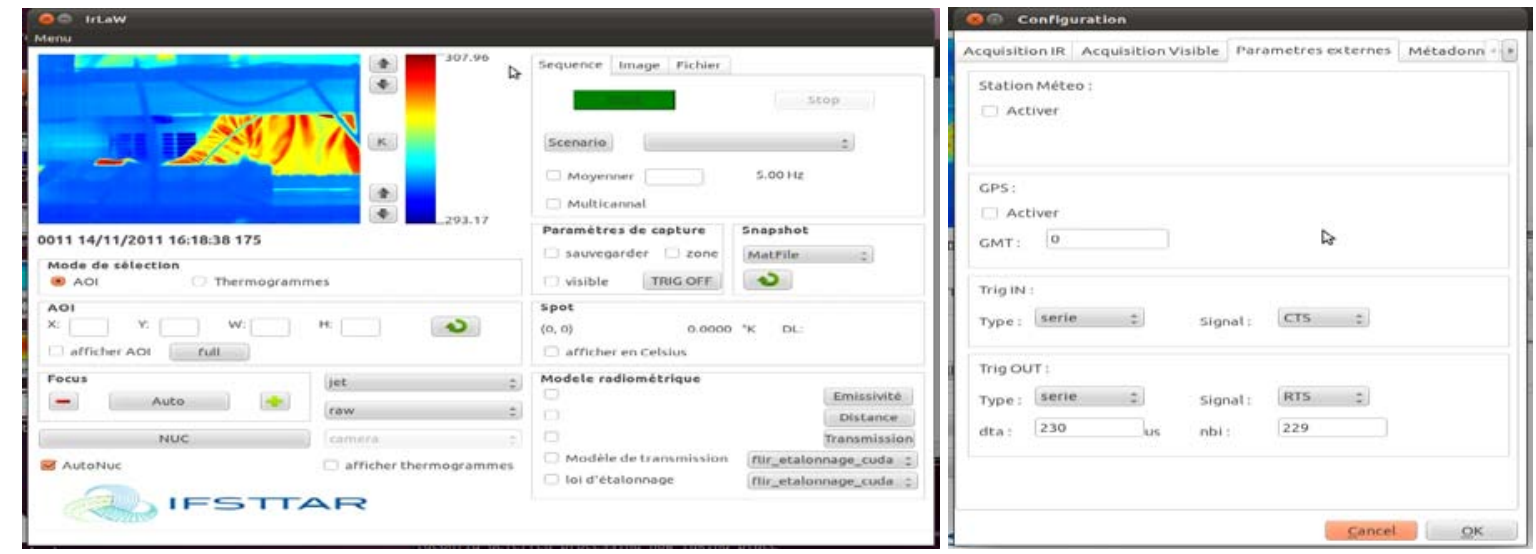

Fig. 5. Screen copies of IrLaw control windows: Main window (left) - Trig, GPS and Weathermeasurement activation (right)

The whole system was tested in laboratory condition and then deployed at field level on real bridges open to traffic during several days in continuous measurement operating conditions.

Next paragraphs present one of the experiments carried out in the framework of the European project ([6], [7]) ISTIMES (Integrated System for Transport Infrastructures surveillance and Monitoring by Electromagnetic Sensing).

\section{System implementation on real site: “Musmeci” bridge experiment}

The whole measurement system was implemented on the "Musmeci" bridge located in Potenza (Italy) in July 2011. No traffic interruption was required during the mounting of our measurement system.

Figure 6 show a schematic representation of the infrared camera implementation on a mast placed on side of the bridge deck. The camera was mounted on top of the mast at $6 \mathrm{~m}$ elevation from the surface of the bridge deck. The weather station was mounted on the same mast at $1 \mathrm{~m}$ under the camera. A GPS antenna was also fixed at the basis of the mast and at a same elevation than the bridge deck surface.

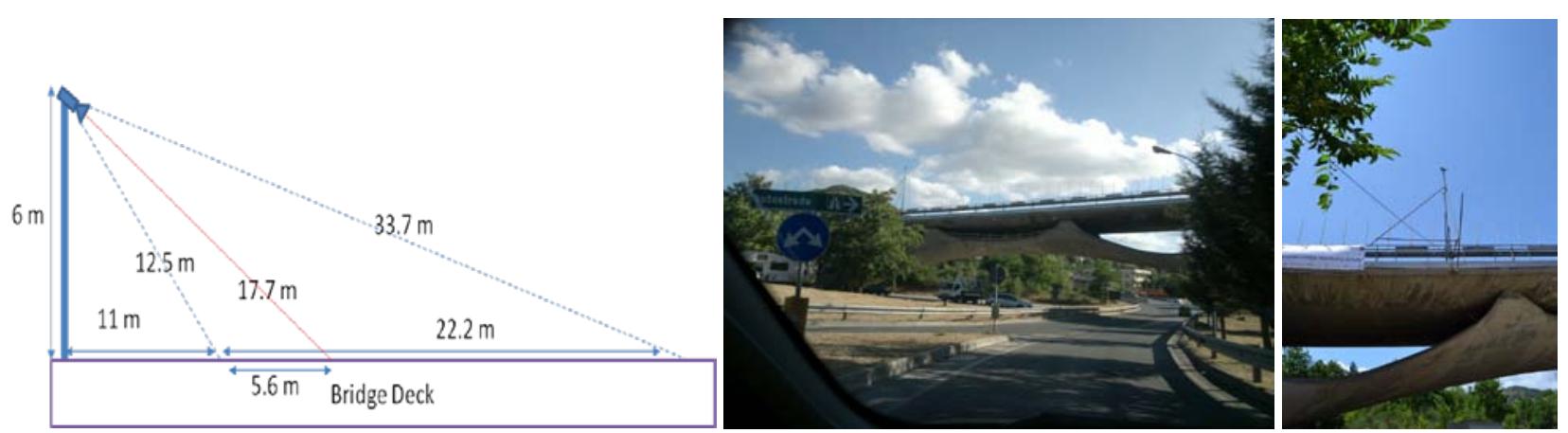

Fig. 6. Schematic representation of the infrared camera on top of "Musmeci"bridge - Pictures of the bridge and of the camera mounted on a mast with a weather station

Figure 7 (right) presents a picture of the bridge deck with a purple area that match the field of view of the infrared camera. Figure 7 (left) presents a screen copy of simulation tools that was used for prototyping the implementation of the infrared camera on the bridge. Measurement of field of view on site using reflective targets and differential GPS measurement (with RTK system) have confirmed the predicted field of view. 

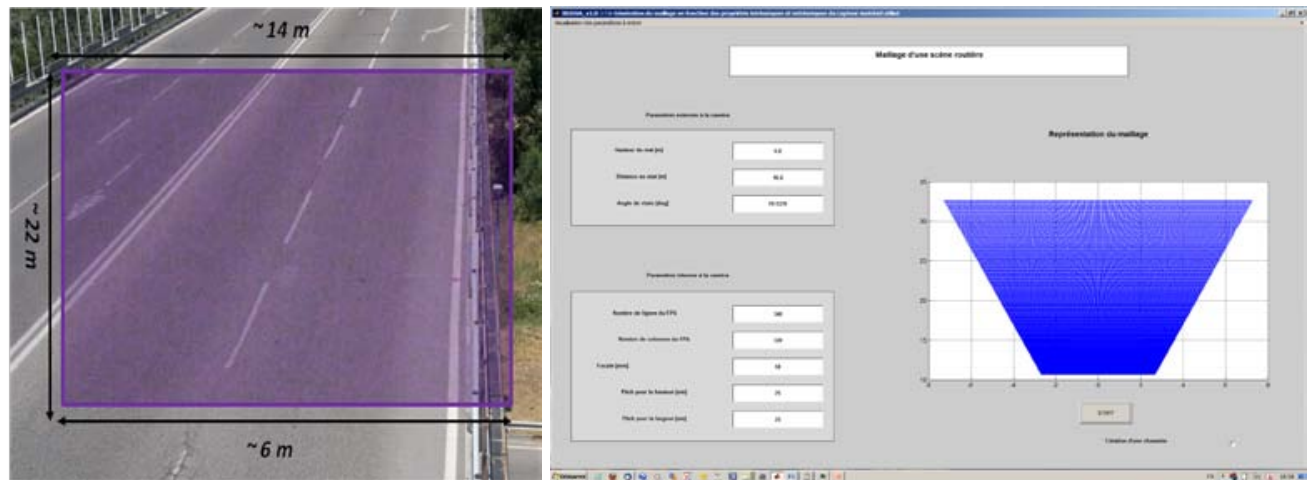

Fig. 7. Schematic representation of the infrared system field of view evaluated at bridge deck level (left) predicted field of view (right) using software IRISSA "InfraRed Image Simulations with Sensor properties Assimilation » dedicated to simplified road scene numerical simulation (see [8])

Figure 8 shows a view of the infrared camera with its protective caisson; the computing (steel blue box) and communication hardware (Access point for wireless remote control); a picture of the bridge deck taken at infrared camera level with the field of view reported in red.

Deployment of the whole prototype system took no more than half working day including system test and settings before measurement launching.
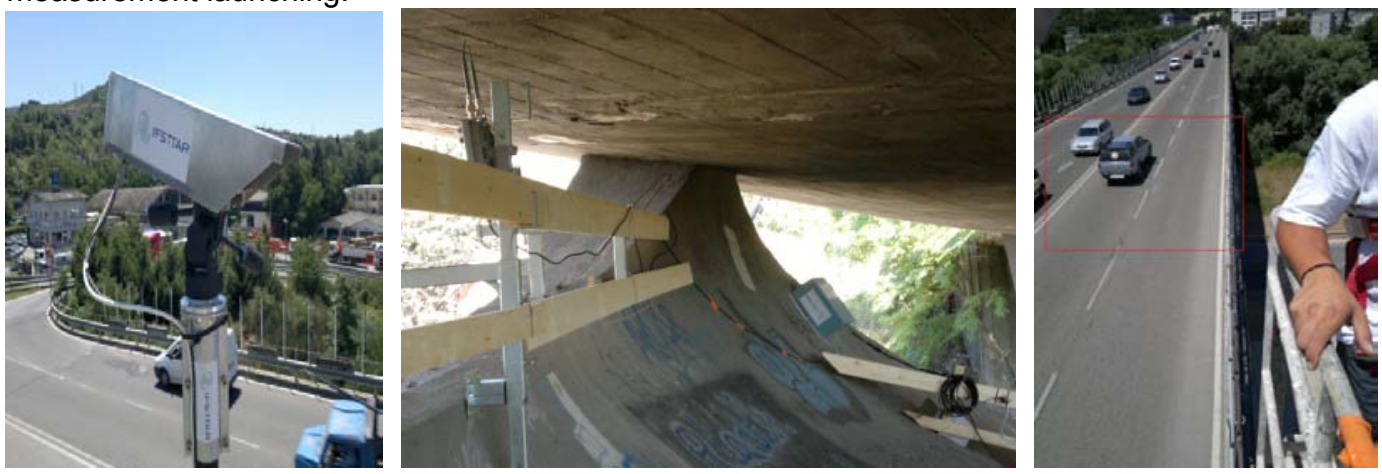

Fig. 8. View of the prototype system deployed on a bridge under traffic - Example of thermal image acquired

Electrical power was provided at bridge level.

\section{Measurement realized and results analysis}

This trial took place during 4 days, but our system was leaved in stand alone acquisition mode only during 3 days. This limitation of time duration for measurements experiments was due to time reserved for assembly and dismantling operations required before and after experiments in order to leave the bridge as it was before experiments. Thanks to the software developed and the small computer hardware used, a thermal image was acquired at a frame rate of $0.1 \mathrm{~Hz}$ by averaging 50 thermal images with camera frame rate fixed at $5 \mathrm{~Hz}$. Each hour, a thermal image sequence was stored on the internal hard drive of our system and data were also retrieved, on demand, by using a wireless connection and a tablet PC.

Figure 9 show 4 thermal images of the bridge deck monitored at different time of the thermal loading and cooling.

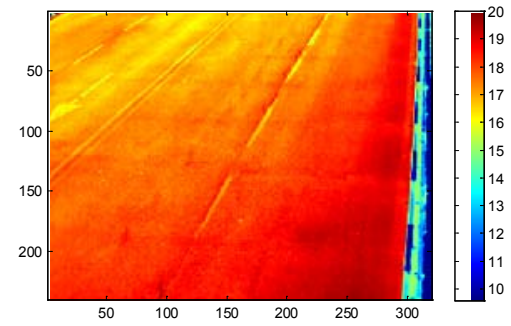

End of thermal relaxation

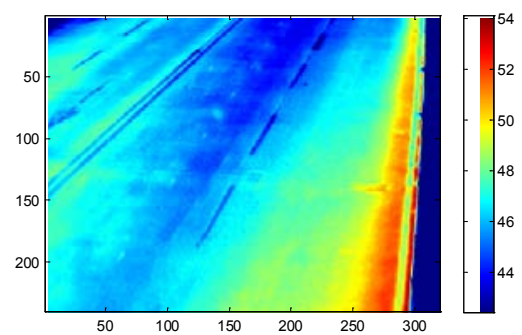

End of temperature raising 


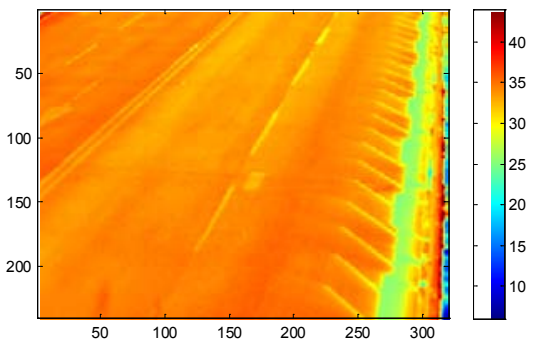

During thermal loading

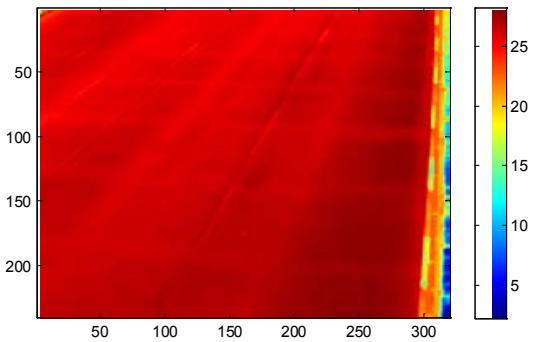

During thermal relaxation

Fig. 9. Example of Thermal images at different moment of the day night cycle (under natural thermal solicitation)

An example of results apparent temperature evolution during experiments is shown on figure 10 (left graph). Thermograms were extracted from thermal image sequence acquired on site during more than 2 days and half, at 3 different locations on the bridge deck surface. Third day of experiment was affected wind gust conditions but also random sun shadowing by clouds. Thermograms are plotted with a time step number for the abscissa axis (two time steps number are separated by 100s). This sampling rate do not favour separation between wind gust effect and direct solar heating shadowing, signal on the third day is noisier. At least two cooling events can be observed on the third day, one at the beginning of the thermal loading and another one round noon. The measured relative humidity (figure 10 right graph) shows burst of humidity increase that match these sudden cooling events, while atmospheric temperature was quite constant due to wind conditions.
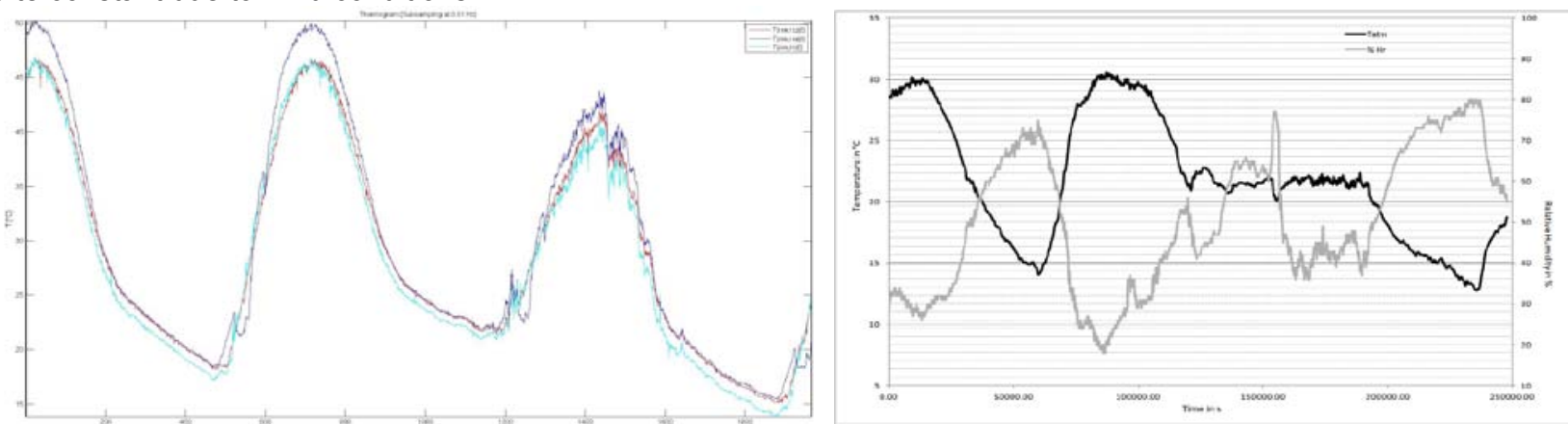

Fig. 10. Apparent temperature evolution measured during round 3 days (left) - Measured atmospheric temperature and relative humidity during the same period (right)

Figure 11 reports the evolution with time of a longitudinal profile and of a transversal profile extracted from thermal images sequence. For longitudinal profile, the slab junction recovered by the pavement wearing course in bitumen concrete could be associated to the different evolution of temperature observed round line 200 on the figure. For transversal profile, non homogeneous spatial distribution of the temperature is observed, it could be linked to sun heating that rise on one side of the bridge and lay down on the other side with a perpendicular illumination at noon.
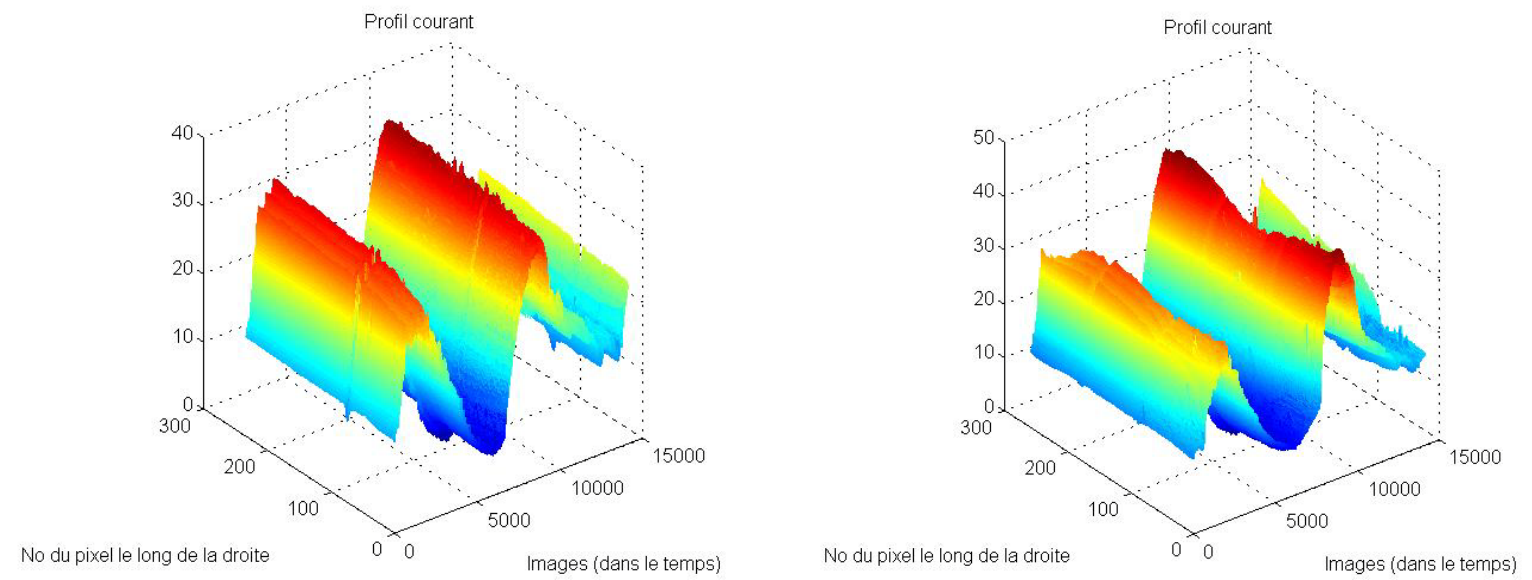

Fig. 11. Longitudinal profile evolution with time (left) - Transversal profile evolution with time (right) 
Frequency analysis approach was applied to thermal image sequence. The Fourier transform (Eq. 2) was applied to temporal evolution of each pixel of the thermal image.

$$
\mathrm{F}_{\mathrm{n}}=\Delta \mathrm{t} \sum_{\mathrm{k}=0}^{\mathrm{N}-1} \mathrm{~T}(\mathrm{k} \Delta \mathrm{t}) \exp ^{(-\mathrm{j} 2 \pi \mathrm{nk} / \mathrm{N})}=R e_{\mathrm{n}}+\operatorname{Im}_{\mathrm{n}}
$$

where $n$ designates the frequency increment $(n=0,1, \ldots N) ; \Delta t$ is the sampling interval; $R e$ and $I m$ are the real and the imaginary parts of the Fourier transform. In practise Fast Fourier Transform algorithm [10] was used to reduce time computing.

Then magnitude $\left(A_{n}\right)$ and phase $\left(\Phi_{n}\right)$ maps were calculated (Eq. 3) and were analysed.

$$
\mathrm{A}_{\mathrm{n}}=\sqrt{\operatorname{Re}_{\mathrm{n}}^{2}+\operatorname{Im}_{\mathrm{n}}^{2}} \text { and } \Phi_{n}=\tan ^{-1}\left(\frac{\operatorname{Im}_{\mathrm{n}}}{R e_{\mathrm{n}}}\right)
$$

Left image in Figure 12 shows a example of Magnitude map computed by FFT calculus [10]where a meshing is observed. This grid match the inner deck structure as presented in middle and right drawing presented in figure 12.

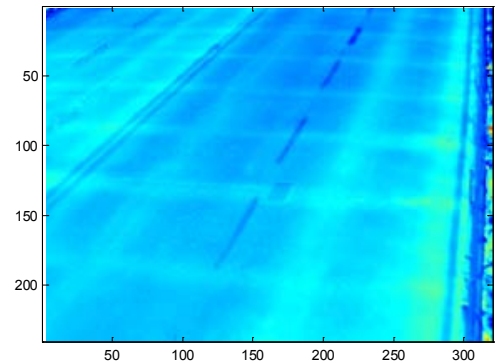

Fig. 12. Example of Magnitude maps computed (left) - Bridge deck cross section (middle) - 3D mesk of the bridge deck structure and pillars (right)

Investigation on inner deck structure were also done by using Singular value decomposition (SVD), allowing to extract the spatial and temporal information from a thermal image sequence in a compact or simplified manner. The SVD of an $M x N$ matrix $\mathbf{A}(M>N)$ can be calculated as follows ([11], [12]):

$$
\mathbf{A}=\mathbf{U R V}^{T}
$$

where $\mathbf{U}$ is an $M x N$ orthogonal matrix, $\mathbf{R}$ being a diagonal $N \times N$ matrix (with the singular values of $\mathbf{A}$ in the diagonal) and $\mathbf{V}^{T}$ is the transpose of an $N x N$ orthogonal matrix (characteristic time).

After rearranging the thermal image for every time as columns in $\mathbf{A}$ and applying the SVD, the columns of $\mathbf{U}$ represent a set of orthogonal statistical modes known as empirical orthogonal functions (EOF) that describes spatial variations of data. On the other hand, the principal components (PCs), which represent time variations, are arranged row-wise in matrix $\mathbf{V}^{T}$. The first EOF will represent the most characteristic variability of the data; the second EOF will contain the second most important variability, and so on. Usually, original data can be adequately represented with only a few EOFs [7-8].

SVD computations were performed on the complete thermal images sequence but also after extraction of a night period in the full sequence (close to linear thermal relaxation). Figure 13 shows EOF map obtained. For full sequence the weight of information concerning the inner structure requires to look farther in EOF maps. For reduced sequence, EOF 2 map give refine sketch of information obtained by full sequence analysis and EOF map though it is affected by traffic signature deliver accurate information on the inner structure (caisson mapping).
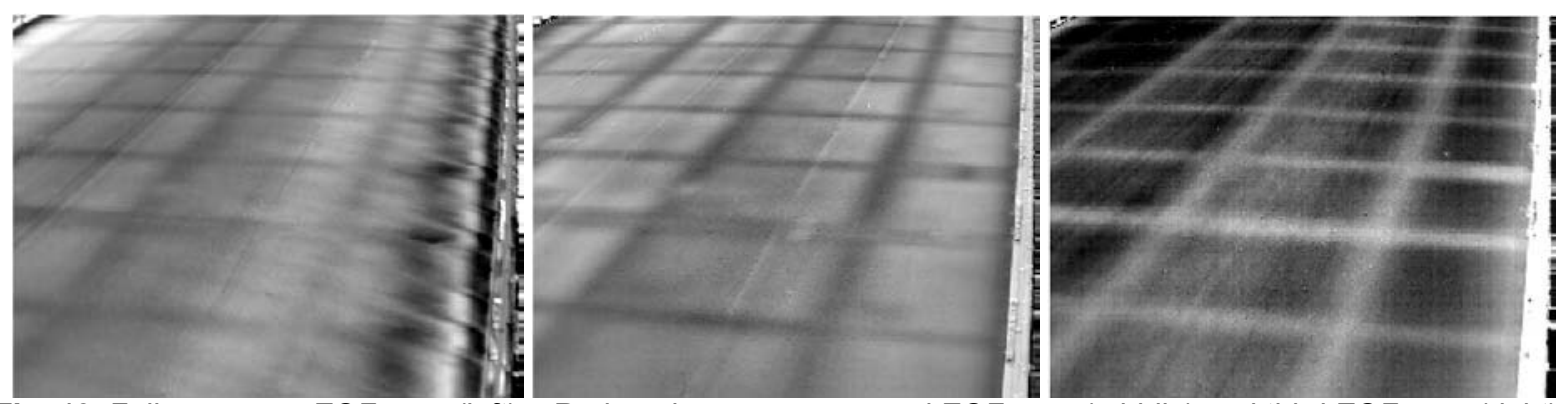

Fig. 13. Full sequence EOF map (left) - Reduced sequence second EOF map (middle) and third EOF map (right) 
Next step will consist in adding Thermal model in the analysis to reconstruct the inner temperature distribution inside the bridge deck and coupling it with mechanical analyis.

\section{Conclusion and Perspectives}

The system studied and developed for long term monitoring of transport infrastructures has been tested on bridges under traffic. "IrLaW" HMI implemented in our measurement plateform was deployed on real bridge for a 3 days monitoring of the apparent surface temperature evolution at a bridge deck level under traffic. The developed application can be remotely controlled both in wire or wireless mode. For field application wireless mode was used and data were stored on board of the platform leaved on site and experimental data were retrieved on demand with a tablet PC. Analysis of results obtained shows that the mesh of inner structure of the bridge deck was retrieved by FFT analysis and with more refinement by using SVD analysis. Extraction of thermal relaxation period was investigated to enhance inner structure detection.

Future works will address complementary correction of infrared measurement by introducing environmental radiative data in the model issued from additional sensors (Pyrgeometer and pyranometer). Furthermore, weather measurements will be analysed inline to allow tagging in thermal image sequence to introduce adapted processing to events observed. Thermal models will be studied taking into account natural environment measurement conditions. We also plan to test self lockin thermography. New functionalities will be added to IrLaw, in particular synchronization capabilities and parallel computing on thermal image streaming by implementing a multi-channel functionality. Finally, experiments will be deployed on real civil engineering elements of transport infrastructure during at least 1 year.

Acknowledgement: The research leading to these results has received funding from the European Community's Seventh Framework Programme (FP7/2007-2013) under Grant Agreement $n^{\circ} 225663$.

\section{REFERENCES}

[1] FLIR systems, "Thermovision A320 et Thermovision A320G: User's Manual”, FLIR A320 camera technical documentation, November 2007.

[2] VAISALA, " Transmetteur météorologique WXT520", guide de l'utilisateur, 2010.

[3] UBLOX, "u-blox 5 Evaluation Kits EVK-5", Technical note, 2009.

[4] NVIDIA, CUDA Toolkit Reference Manual, 2010 (www.nvidia.com).

[5] G. Gaussorgues, "La thermographie infrarouge : Principes, Thechnologies, Applications", Tec Doc, Lavoisier, $3^{\mathrm{e}}$ édition, 1989.

[6] ISTIMES www.istimes.eu

[7] Proto, M., Bavusi, M., Bernini, R., Bigagli, L., Bost, M., Bourquin, F., Cottineau, L.-M., Cuomo, V., Vecchia, P.D., Dolce, M., Dumoulin, J., Eppelbaum, L., Fornaro, C., Gustafsson, M., Hugenschimdt, J., Kaspersen, P., Kim, H., Lapenna, V., Leggio, M., Loperte, A., Mazzetti, P., Moroni, C., Nativi, S., Nordebo, S., Pacini, F., Palombo, A., Pascucci, S., Perrone, A., Pignatti, S., Ponzo, F.C., Rizzo, E., Soldovieri, F. and Taillade, F., 2010. Transport infrastructure surveillance and monitoring by electromagnetic sensing: the ISTIMES project. Sensors, 10, 10620-10639, doi: 10.3390/s101210620.

[8] J. Dumoulin, F. Delannay et G. Lecamus, « Enhancement of infrared vision for drivers by acting on infrastructure », TRA 2006 (Transportation Research Arena), Göteborg, Suède, 12-15 juin 2006.

[9] E. P. SHETTLE et R. W. FENN., "Models for the aerosols of the lower atmosphere and the effects of humidity variations on their optical properties", Air Force Geophysics Laboratory 79-0214, (1979).

[10] Cooley J.W., Tukey J.W., "An algorithm for the machine calculation of complex Fourier series", Mathematics of Computation, vol. 19, n 90, 1965, p. 297-301.

[11] Rajic N., "Principal component thermography for flaw contrast enhancement and flaw depth characterization in composite structures", Composite Structures, vol 58, pp 521-528, 2002.

[12] Marinetti S., Grinzato E., Bison P. G., Bozzi E., Chimenti M., Pieri G. And Salvetti O. "Statistical analysis of IR thermographic sequences by PCA," Infrared Physics \& Technology vol 46 pp 85-91, 2004. 